\title{
Invariants of Orthogonal G-Modules from the Character Table
}

\author{
Gabriele Nebe
}

\section{CONTENTS}

1. Introduction

2. Clifford Algebras

3. Clifford Algebras as G-Algebras

4. Examples

Acknowledgments

References
Character-theoretic methods using Clifford algebras are developed to describe the quadratic forms on a vector space $V$ that are invariant under a finite subgroup $G$ of $G L(V)$ such that the order of the commutator factor group of $\mathrm{G}$ is odd.

\section{INTRODUCTION}

Let $G$ be a finite group. Any $\mathbb{Q} G$-module $V$ is uniquely determined by its character $\chi_{V}$. So in principle $\chi_{V}$ also determines the $G$-invariant quadratic forms on $V$. However, there is not much known how to calculate rational invariants of these quadratic forms without describing the action of $G$ on $V$ explicitly. Of course, the $G$-invariant form $q$ on $V$ is not unique, since for all $a \in \mathbb{Q}$, the form $a q$ is also $G$-invariant, and there are more $G$-invariant forms, if $V$ is not irreducible over the reals. So one can only hope to calculate say the determinant of $q$, which is defined as the determinant of a Gram matrix of the corresponding bilinear form modulo squares, if $\operatorname{dim} V$ is even.

Invariants for a nondegenerate quadratic space $\varphi:=(V, q)$ over an arbitrary field $K$ can be read off from the Clifford algebra $C(\varphi)$. This is a $\mathbb{Z} / 2 \mathbb{Z}$ graded algebra functorially attached to $\varphi$ and it determines the two most important invariants of the quadratic space $\varphi$ : its determinant and its Clifford invariant (see Theorem 2.1). According to [Hasse 1924], if $V$ is a vector space over a number field $K$, then these two invariants together with the dimension of $V$ and the signatures of $\varphi$ at all real places of $K$ describe the $K$-isometry class of the nondegenerate quadratic space $\varphi$ over $K$ completely.

If $G$ acts on $\varphi=(V, q)$ as isometries, then $G$ acts on $C(\varphi)$ as algebra automorphisms respecting the grading. If $\operatorname{dim} V$ is even, $C(\varphi)=: c(\varphi)$ is central 
simple; if $\operatorname{dim} V$ is odd, the even part $c(\varphi):=C_{0}(\varphi)$ of the Clifford algebra is a central simple algebra. So the simple $c(\varphi)$-module $W$ becomes a module for some projective representation of $G$ (in the sense of Schur) of which the character $\chi_{W}$ (of a covering group of $G$ ) is a certain square root of a character constructed from $\chi_{V}$. An irreducible constituent that occurs in $\chi_{W}$ with odd multiplicity gives information on either the determinant of $\varphi$, if $\operatorname{dim} V$ is even, or the Clifford invariant of $\varphi$, if $\operatorname{dim} V$ is odd (see Corollary 3.6). Applications are given in the examples in Section 4.

Related ideas have been used by A. Turull [1992], where he determines the Schur index of $\chi_{W}$ using explicit knowledge about $(V, q)$ for the $(n-1)$-dimensional $S_{n}$-module $V$.

This paper summarizes one idea of my Habilitationsschrift [Nebe 1999]. There I develop also other methods to calculate the isometry class of $\varphi$ using constructions of $V$ as a constituent of a tensor product or of an induced module. See also [Nebe 2000].

\section{CLIFFORD ALGEBRAS}

Let $\varphi:=(V, q)$ be a quadratic space of dimension $n:=\operatorname{dim} V$ over a field $K$. Then the Clifford algebra $C(\varphi):=T(V) / I(\varphi)$ is the quotient of the tensor algebra $T(V):=\bigoplus_{i=0}^{\infty} \otimes^{i} V$ by the two-sided ideal $I(\varphi)$ generated by $v \otimes v-q(v) \cdot 1$ with $v \in V$ (see [Scharlau 1985, Chapter 9]). $C(\varphi)$ is a $2^{n_{-}}$ dimensional $K$-algebra. It contains the even Clifford algebra

$$
\left.C_{0}(\varphi):=\left\langle v_{1} \ldots v_{s}\right| v_{i} \in V, s \text { even }\right\rangle \leq C(\varphi)
$$

as a subalgebra of dimension $2^{n-1}=\operatorname{dim}_{K}\left(C_{0}(\varphi)\right)$. Let

$$
\left.C_{1}(\varphi):=\left\langle v_{1} \ldots v_{s}\right| v_{i} \in V, s \text { odd }\right\rangle
$$

If $\left(v_{1}, \ldots, v_{n}\right)$ is a basis of $V$, then $\left(v_{i_{1}} \ldots v_{i_{s}} \mid 1 \leq\right.$ $\left.i_{1}<\cdots<i_{s} \leq n\right)$ is a basis of $C(\varphi)$. In particular, $V$ is embedded in $C(\varphi)$.

If char $K \neq 2$, then let $B_{q}$ be the bilinear form associated to $q$, defined by

$$
B_{q}(v, w):=\frac{1}{2}(q(v+w)-q(v)-q(w))
$$

for all $v, w \in V$. Then the determinant $\operatorname{det}(\varphi)$ is the determinant of a Gram matrix of $B_{q}$ modulo squares

$$
\operatorname{det}(\varphi):=\operatorname{det}\left(B_{q}\left(v_{i}, v_{j}\right)\right)_{i, j=1}^{n}\left(K^{*}\right)^{2} \in K /\left(K^{*}\right)^{2}
$$

and the discriminant of $\varphi$ is

$$
d_{ \pm}(\varphi)=(-1)^{\left(\begin{array}{l}
n \\
2
\end{array}\right)} \operatorname{det}(\varphi) \in K /\left(K^{*}\right)^{2} .
$$

Assume that char $K \neq 2$ and $\varphi$ is nondegenerate, that is, $d_{ \pm}(\varphi) \neq 0$. Then:

Theorem 2.1 [Scharlau 1985, Theorem 9.2.10]. (i) If n is even, then $C(\varphi)$ is a central simple $K$-algebra and $Z\left(C_{0}(\varphi)\right)$ is isomorphic to

$$
K[X] /\left(X^{2}-d_{ \pm}(\varphi)\right) .
$$

(ii) If $n$ is odd, then $C_{0}(\varphi)$ is a central simple $K$ algebra and $Z(C(\varphi))$ is isomorphic to

$$
K[X] /\left(X^{2}-d_{ \pm}(\varphi)\right) .
$$

An analogous theorem holds if char $K=2$; see [Knus 1991, Theorem (2.2.3)].

Let

$$
c(\varphi):= \begin{cases}C(\varphi) & \text { if } \operatorname{dim} V \text { is even, } \\ C_{0}(\varphi) & \text { if } \operatorname{dim} V \text { is odd }\end{cases}
$$

The class $[c(\varphi)] \in \operatorname{Br} K$ of the central simple $K$ algebra $c(\varphi)$ in the Brauer group $\operatorname{Br} K$ of $K$ is called the Clifford invariant of $\varphi$.

Remarks 2.2. (i) If $W$ is the simple right $c(\varphi)$-module, $D:=\operatorname{End}_{c(\varphi)}(W)$, and $W^{*}:=\operatorname{Hom}_{D}(W, D)$ is the simple left $c(\varphi)$-module, then $c(\varphi) \cong W^{*} \otimes_{D}$ $W$ as $c(\varphi)$-bimodule.

(ii) Because of the universal property of $C(\varphi)$, the identity embedding of $V \subset C(\varphi)$ extends to a unique algebra antiautomorphism of $C(\varphi)$, the canonical involution - . The involution satisfies $\overline{v_{1} \ldots v_{s}}=v_{s} \ldots v_{1}$ for all $v_{i} \in V$ and induces an algebra antiautomorphism on the central simple $K$-algebra $c(\varphi)$. Via this involution $W^{*}$ becomes a right $c(\varphi)$-module isomorphic to $W$.

\section{The Orthogonal Group}

For a nondegenerate quadratic space $\varphi=(V, q)$ the orthogonal group $O(\varphi)$ is defined as

$O(\varphi):=\{f \in \mathrm{GL}(V) \mid q(f(v))=q(v)$ for all $v \in V\}$.

Using the universal property of $C(\varphi)$ one easily sees that the linear action of $O(\varphi)$ on $V$ extends uniquely to $K$-algebra automorphisms of the Clifford algebra $C(\varphi)$. This action of $O(\varphi)$ on $C(\varphi)$ respects the grading.

The Clifford group is

$\Gamma(\varphi):=\left\{s \in C_{0}(\varphi)^{*} \cup\left(C(\varphi)^{*} \cap C_{1}(\varphi)\right) \mid s V s^{-1}=V\right\}$ 
[Scharlau 1985, Section 9.3; Knus 1991, Section IV.6]. For $s \in \Gamma(\varphi)$ let $\gamma(s)=1$ if $s \in C_{0}(\varphi)$ and $\gamma(s)=-1$ if $s \in C_{1}(\varphi)$. Define $\alpha(s): V \rightarrow V$ so that

$$
\alpha(s)(v) \gamma(s) s^{-1} v s .
$$

Then $\alpha(\Gamma(\varphi)) \subset O(\varphi)$. If $s \in V \subset C_{1}(\varphi)$ satisfies $q(s) \neq 0$, then $s \in \Gamma(\varphi)$ and $\alpha(s) \in O(\varphi)$ is the reflection along the anisotropic vector $s$. If char $K \neq$ 2 , then the reflections along anisotropic vectors in $V$ generate $O(\varphi)$. Therefore $\alpha(\Gamma(\varphi))=O(\varphi)$. If char $K=2$ then $\alpha$ is also surjective [Knus 1991, p. 228]. The kernel of $\alpha$ is $K^{*} \subset C_{0}(\varphi)^{*}$ and one has the following exact sequence

$$
1 \rightarrow K^{*} \rightarrow \Gamma(\varphi) \rightarrow O(\varphi) \rightarrow 1
$$

which gives rise to a projective representation

$$
P: O(\varphi) \rightarrow \Gamma(\varphi)
$$

mapping the reflection along the anisotropic vector $s \in V$ to $s \in \Gamma(\varphi)$. By [Scharlau 1985, Lemma 9.3.2], $s \bar{s} \in K^{*} \subseteq C_{0}(\varphi)$ for all $s \in \Gamma(\varphi)$, where ${ }^{-}$ is the canonical involution of $c(\varphi)$ (see Remark 2.2). This gives rise to a group homomorphism

$$
\text { norm : } \begin{aligned}
O(\varphi) & \rightarrow K^{*} /\left(K^{*}\right)^{2}, \\
g & \mapsto P(g) \cdot \overline{P(g)} \cdot\left(K^{*}\right)^{2},
\end{aligned}
$$

called the Spinor norm [Scharlau 1985, Definition 9.3.4]. Let

$$
S \Gamma(\varphi):=\left\{s \in \Gamma(\varphi) \cap C_{0}(\varphi) \mid \bar{s}=s^{-1}\right\} .
$$

Proposition 2.3. The group $S \Gamma(\varphi)$ acts on $c(\varphi)$ by conjugation: $c(\varphi) \times S \Gamma(\varphi) \rightarrow c(\varphi),(x, s) \mapsto s^{-1} x s$. With the notations of Remark 2.2 one has

$$
c(\varphi) \cong W \otimes_{D} W
$$

as $S \Gamma(\varphi)$-modules.

\section{CLIFFORD ALGEBRAS AS G-ALGEBRAS.}

Let $\varphi=(V, q)$ be a nondegenerate quadratic space and $G$ be a subgroup of the orthogonal group $O(\varphi)$. Then $\varphi$ is also called an orthogonal KG-module. Theoretical concepts for Clifford algebras as $G$-algebras are given in [Fröhlich 1972]. Here practical methods to obtain information on $C(\varphi)$ from the character $\chi=\chi_{V}$ of the $G$-module $V$ are developed.

Since $G \leq O(\varphi)$, the action of $O(\varphi)$ on $C(\varphi)$ restricts to a linear representation $\Delta_{C(\varphi)}$ of $G$ on the Clifford algebra that respects the grading:
Remark 3.1. The character of the $K G$-module $C(\varphi)$ respectively $C_{0}(\varphi)$ is

$$
\tilde{\chi}:=\sum_{i=0}^{n} \Lambda^{i}(\chi) \text { respectively } \tilde{\chi}_{0}:=\sum_{i=0, i \text { even }}^{n} \Lambda^{i}(\chi)
$$

where $\Lambda^{i}(\chi)$ is the $i$-th exterior power of $\chi$.

Note that

$$
\tilde{\chi}(g)=(-1)^{n} p_{g}(-1) \text { for all } g \in G
$$

where $p_{g}$ is the characteristic polynomial of $g$ on $V$. With this trick one can calculate $\tilde{\chi}(g)$ (and $\tilde{\chi}_{0}(g)$ ) with the help of GAP [Schönert et al. 1994], by restricting $\chi$ to the subgroup $\langle g\rangle \leq G$, for any group $G$ whose character table and powermap is known.

Assumption. From now on we assume that the order of the commutator factor group $G / G^{\prime}$ is odd.

Lemma 3.2. There are $a_{g} \in K^{*}$ (for all $g \in G$ ) such that $P_{0}(g):=a_{g} P(g)$ satisfies

$$
P_{0}(g) \subseteq C_{0}(\varphi), \quad P_{0}(g) \overline{P_{0}(g)}=1,
$$

and $P_{0} \otimes P_{0}: G \rightarrow \mathrm{GL}(c(\varphi))$ is a linear representation equivalent to $\Delta_{c(\varphi)}$.

Proof. The mapping $P: G \rightarrow C(\varphi)^{*}, g \mapsto P(g)$ is a projective representation of $G$. Hence $P(g) P(h)=$ $a(g, h) P(g h)$ for some $a(g, h) \in K^{*}$ and for all $h, g \in$ $G$. Since $K^{*} \subseteq C_{0}(\varphi)$, the grading of the Clifford algebra defines a group homomorphism $G \rightarrow \mathbb{Z} / 2 \mathbb{Z}$, $g \mapsto \operatorname{deg}(P(g))$. Since $\left|G / G^{\prime}\right|$ is odd, this group homomorphism is trivial, hence $P(G) \subseteq C_{0}(\varphi)$. In particular $P(G) \subseteq c(\varphi)$ and $P$ is a projective representation of $G$ on the simple $c(\varphi)$-module $W$. Also the Spinor norm, norm : $G \rightarrow K^{*} /\left(K^{*}\right)^{2}$, is a homomorphism from $G$ to an abelian 2-group and hence trivial. Rescaling $P(g)$ with elements in $K^{*}$ we may therefore assume that $P(g) \overline{P(g)}=1$, that is, $P(g) \in S \Gamma(\varphi)$ for all $g \in G$.

Therefore, by Proposition 2.3, the $G$-module $c(\varphi)$ is isomorphic to the tensor square $W \otimes_{D} W$ of the projective $G$-module $W$. In particular the projective representation $P \otimes_{D} P$ is equivalent (as a projective representation) to the linear representation $\Delta_{c(\varphi)}$ of $G$ on $c(\varphi)$. This means that there is $T \in \operatorname{GL}(c(\varphi))$ and a mapping $\alpha: G \rightarrow K^{*}$ such that $T P(g) \otimes$ $P(g) T^{-1}=\alpha(g) \Delta_{c(\varphi)}(g)$ for all $g \in G$. Since $P$ is a projective representation and $\Delta_{c(\varphi)}$ is linear, $\alpha$ : $G \rightarrow K^{*} /\left(K^{*}\right)^{2}$ is a homomorphism. Again, since 
$\left|G / G^{\prime}\right|$ is odd, we have $\alpha(g)=\beta(g)^{2} \in\left(K^{*}\right)^{2}$ for all $g \in G$ and $P_{0}(g):=\beta(g)^{-1} P(g)$ has the desired properties.

Corollary 3.3 (compare [Gagola and Garrison 1982, Theorem 1.2, Corollary 4.3]). Assume that char $K \neq$ 2 and let $g \in G$ be an element of order 2 and $e$ the dimension of the-1-eigenspace of $g$ in $V$. Then $P_{0}(g)^{2}=(-1)^{\left(\begin{array}{l}e \\ 2\end{array}\right)}$ id.

Proof. Let $v_{1}, \ldots, v_{e}$ be an orthogonal basis of the -1-eigenspace of $g$ on $V$. Then

$$
\begin{aligned}
& P_{0}(g)=a_{g} v_{1} \ldots v_{e}, \\
& \overline{P_{0}(g)}=a_{g} v_{e} \ldots v_{1}=(-1)^{\left(\begin{array}{l}
e \\
2
\end{array}\right)} P_{0}(g) .
\end{aligned}
$$

Since $P_{0}(g) \overline{P_{0}(g)}=\mathrm{id}$, one has $P_{0}(g)^{2}=(-1)^{\left(\begin{array}{l}e \\ 2\end{array}\right)}$ id.

If $c(\varphi) \cong D^{a \times a}$ for some central $K$-division algebra $D$, then the simple $c(\varphi)$-module $W$ is isomorphic to $D^{a}$. Over the algebraic closure of $K$, the $c(\varphi)$ module $W$ is isomorphic to the sum of $m$ copies of a simple module, where $m$ is the index of $D$ $\left(\operatorname{dim}_{K}(D)=m^{2}\right)$.

We now fix a covering group $u: \tilde{G} \rightarrow G$ of $G$ such that $P_{0}$ is equivalent to a linear representation of $\tilde{G}$. Let $W$ be the simple $c(\varphi)$-module and $m$ the index of $\operatorname{End}_{c(\varphi)}(W)$.

Corollary 3.4. Let $m \chi_{W}$ be the character of a linear $K \tilde{G}$-module that is equivalent to $W$ over the algebraic closure of $K$. Regarding $\chi$ as a character of $\tilde{G}$ one has

$$
\chi_{W} \otimes \chi_{W}= \begin{cases}\tilde{\chi} & \text { if } n \text { is even }, \\ \tilde{\chi}_{0} & \text { if } n \text { is odd. }\end{cases}
$$

For the next theorem we additionally assume that $K$ is a number field. By [Scharlau 1985, Lemma 9.2.8] $c(\varphi)$ is a tensor product of quaternion algebras. Since $K$ is a number field, this implies that $c(\varphi)$ is a matrix ring over a quaternion algebra (see [Reiner 1975, Theorem (32.9)], for instance) and

$$
C_{0}(\varphi) \cong D^{a \times a}
$$

where $D=L:=Z\left(C_{0}(\varphi)\right)$ or $D$ is a quaternion division algebra over $L$.

Theorem 3.5. With the notations above let $m$ be the Schur index of $D, W$ the simple $C_{0}(\varphi)$-module and $m \chi_{W}$ the corresponding character of $\tilde{G}$. Assume that there is an absolutely irreducible character $\psi$ of $\tilde{G}$ occurring with odd multiplicity in $\chi_{W}$.

(a) If $n$ is even and $L=Z\left(C_{0}(\varphi)\right)$ is a field then $L$ is a subfield of the character field $K(\psi)$.

(b) Assume that $n$ is odd. If the Schur index of $\psi$ is odd, then $K(\psi)$ splits $D$. Otherwise let $U$ be the irreducible $K \tilde{G}$-module whose character contains $\psi$. Then $D \subset \operatorname{End}_{K \tilde{G}}(U)$.

Proof. By Lemma 3.2 $P(G)$ is already contained in $C_{0}(\varphi)$ and therefore $\operatorname{End}_{C_{0}(\varphi)}(W) \subseteq \operatorname{End}_{\tilde{G}}(W)$.

In both cases $C_{0}(\varphi) \cong D^{a \times a}$ and $\operatorname{dim}_{K}\left(C_{0}(\varphi)\right)=$ $a^{2} m^{2}[L: K]=2^{n-1}$ is a power of 2 . Let $x$ be the multiplicity of $\psi$ in $\chi_{W}, U$ the irreducible $K$-module whose character contains $\psi$ and $D_{U}:=\operatorname{End}_{K \tilde{G}}(U)$. Then $D_{U}$ is a skew field with center $K(\psi)$ and of index, say, $m_{U}$. Let $U^{\prime}$ be the $U$-homogeneous component in $W_{\mid \tilde{G}}$. Then $\operatorname{End}_{K \tilde{G}}\left(U^{\prime}\right) \cong D_{U}^{y \times y}$ for some $y \in \mathbb{N}$. Since the multiplicity of $\psi$ in $m \chi_{W}$ and the multiplicity of $\psi$ in $\chi_{U^{\prime}}$ are equal, one has

$$
m x=y m_{U} .
$$

Since $D$ has no zero divisors, $D$ embeds into

$$
\operatorname{End}_{K \tilde{G}}\left(U^{\prime}\right)
$$

and hence

$$
\begin{aligned}
A:=D_{U}^{o p} & \otimes_{K(\psi)}\left(D \otimes_{K} K(\psi)\right) \\
& \rightarrow D_{U}^{o p} \otimes_{K(\psi)} D_{U}^{y \times y} \cong K(\psi)^{m_{U}^{2} y \times m_{U}^{2} y}=: B,
\end{aligned}
$$

where $D_{U}^{o p}$ denotes the opposite algebra of $D_{U}$. If $K(\psi) \otimes_{K} L$ is a field then let $\varepsilon:=[L: K] \in\{1,2\}$. Then $A$ is a central simple $K(\psi) \otimes_{K} L$-algebra isomorphic to $\tilde{D}^{k \times k}$ for some central $K(\psi) \otimes_{K} L$-division algebra $\tilde{D}$ of index, say, $\tilde{m}$. If $K(\psi) \otimes_{K} L$ is not a field then let $\varepsilon:=1$. Then $A$ is a direct sum of two isomorphic central simple $K(\psi)$-algebras isomorphic to $\tilde{D}^{k \times k}$ for some central $K(\psi)$-division algebra $\tilde{D}$ of index, say, $\tilde{m}$.

In both cases the dimension of $A$ over its center is

$$
m_{U}^{2} \cdot m^{2}=\tilde{m}^{2} \cdot k^{2}
$$

and the $K(\psi)$-dimension of a simple $A$-module is $\varepsilon \cdot \tilde{m}^{2} \cdot k$ and divides the $K(\psi)$-dimension of the simple $B$-module, which is $m_{U}^{2} \cdot y$

$$
\varepsilon \cdot \tilde{m}^{2} \cdot k \text { divides } m_{U}^{2} \cdot y \text {. }
$$

Claim. $\tilde{m}$ is odd and $\varepsilon=1$. 
Proof. Since $K$ is a number field, $m$ is either 1 or 2 . If $m=1$, then $m_{U}$ and $y$ are odd by (3-1) (recall that $x$ is odd) and hence also $\tilde{m}$ and $\varepsilon$ are odd. Assume that $m=2$. Then $(3-1)$ implies that either $m_{U}$ is even and $y \cdot m_{U} / 2$ is odd, or $y$ is even and $m_{U} \cdot y / 2$ is odd. Assume $2 \mid \tilde{m}$. If $m_{U}$ is even, $2^{3}$ divides $\tilde{m}^{2} \cdot k$; if $m_{U}$ is odd, $2^{2}$ divides $\tilde{m}^{2} \cdot k$. But this power of 2 does not divide $m_{U}^{2} \cdot y$ in both cases, which is a contradiction. Therefore $\tilde{m}$ is odd and $k$ is even. If $m_{U}$ is even, then 4 divides $k$ by (3-2) and therefore $\varepsilon$ is odd. If $m_{U}$ is odd, then also $\varepsilon=1$ since $k$ is even and $y / 2$ is odd. The claim follows.

In particular $\varepsilon=1$ and hence $L$ is a subfield of $K(\psi)$ which proves (a).

Now we prove (b). Since $n$ is odd, $L=K$ and $\left(\left[D_{U}\right]^{-1} \cdot\left[D \otimes_{K} K(\psi)\right]\right)$ has odd order in the Brauer group of $K(\psi)$ because

$$
\left(\left[D_{U}\right]^{-1} \cdot\left[D \otimes_{K} K(\psi)\right]\right)^{\tilde{m}}=1 \in \operatorname{Br}(K(\psi))
$$

Therefore the local index $m_{\wp}\left(D_{U}\right)$ is even, if and only if the local index $m_{\wp}\left(D \otimes_{K} K(\psi)\right)$ is 2, for all (infinite and finite) places $\wp$ of $K(\psi)$. Hence $D \otimes_{K} K(\psi)$ embeds into $D_{U}$ (compare [Reiner 1975, Exercise 29.7]).

In the applications absolutely irreducible orthogonal $G$-modules $(V, q)$ over totally real number fields $K$ are of special interest. Then $q$ is (positive or negative) definite. If $n$ is even, then the discriminant of $q$ is negative, if $n \equiv 2(\bmod 4)$ and positive, if $4 \mid n$.

Corollary 3.6. In addition to the assumptions of the theorem let $K$ be a totally real number field and assume that $\varphi$ is definite.

(a) Let $n$ be even. If $[K(\psi): K]$ is odd or $n \equiv 0$ $(\bmod 4)$ and all intermediate fields $K(\psi) \supset L \supset$ $K$ of degree $[L: K]=2$ are complex fields, then the discriminant $d_{ \pm}(\varphi)=1$.

If $n \equiv 2(\bmod 4)$, then $K(\psi) / K$ has a totally complex intermediate field $L$ with $[L: K]=2$. One of these fields is isomorphic $K\left[\sqrt{d_{ \pm}(\varphi)}\right]$.

(b) Assume that $n$ is odd. If $\psi$ has Schur index 1 , then the Clifford invariant $[c(\varphi)]$ satisfies

$$
\left[c(\varphi) \otimes_{K} K(\psi)\right]=[K(\psi)] \in \operatorname{Br}_{2}(K(\psi)) .
$$

If $\psi$ has Schur index 2 then $\left[K(\psi) \otimes_{K} c(\varphi)\right]=$ $\left[\operatorname{End}_{K(\psi) \tilde{G}}(U)\right] \in \operatorname{Br}_{2}(K(\psi))$ for the irreducible $K(\psi) G$-module $U$ with character $2 \psi$.

\section{EXAMPLES}

We will now apply the methods presented before to some irreducible representations of finite quasi simple groups. The notations are taken from [Conway et al. 1985].

\section{Example 1}

Let $G \cong 2 . O_{8}^{+}(2)$. Then $G$ is perfect and its universal covering group is $\tilde{G} \cong 2^{2} \cdot O_{8}^{+}(2)$. Let $V$ be the 8-dimensional faithful $\mathbb{Q} G$-module with character $\chi$ and $q$ a non zero $G$-invariant quadratic form on $V$. If $\varphi:=(V, q)$, then $\operatorname{dim}(c(\varphi))=2^{8}$ and $\tilde{\chi}=\chi_{W} \otimes \chi_{W}$ for a 16 -dimensional $\tilde{G}$-module $W$. One calculates that $\chi_{W}=\chi_{8}+\chi_{8}^{\prime}$ is the sum of the two irreducible characters $\chi_{8}, \chi_{8}^{\prime} \neq \chi$ which belong to absolutely irreducible rational modules of degree 8 of $\tilde{G}$. Therefore $d_{ \pm}(\varphi)=1$ and also $[c(\varphi)]=[\mathbb{Q}]$.

Of course, that $d_{ \pm}(\varphi)=1$ is well known and can also be seen by inspection of the modular constituents of $V$ [Jansen et al. 1995].

\section{Example 2}

Let $G \cong M^{c} L$ and $\varphi:=(V, q)$ a 22-dimensional orthogonal $\mathbb{Q} G$-module with character $\chi$. The universal covering group of $G$ is 3.G. Therefore $P_{0}$ : $G \rightarrow c(\varphi)$ can be chosen to be linear. There is a unique character $\chi_{W}$ of $G$ satisfying $\chi_{W} \otimes \chi_{W}=\tilde{\chi}$. In the notation of [Conway et al. 1985] one has $\chi_{W}=2\left(\chi_{1}+\chi_{2}+\chi_{3}\right)+\chi_{5}+\chi_{6}$. Now the character field $\mathbb{Q}\left[\chi_{5}\right]=\mathbb{Q}\left[\chi_{6}\right]=\mathbb{Q}[\sqrt{-15}]$. Since $\operatorname{dim}(V) \equiv 2$ $(\bmod 4)$, Corollary 3.6 yields $d_{ \pm}(\varphi)=-15$.

\section{Example 3}

Let $G \cong S_{6}(3)$ and $\chi$ the irreducible character of degree 78 with orthogonal $\mathbb{Q} G$-module $\varphi:=(V, q)$. The universal covering group of $G$ is $\tilde{G} \cong 2 . S_{6}(3)$. Let $\chi_{W}$ be the character of $\tilde{G}$ on the simple $c(\varphi)$ module. If $g \in G$ is an element of order 2 in class $2 B$ in the notation of [Conway et al. 1985], then $-g$ has a 42-dimensional fixed space on $V$. Therefore $\chi_{W}$ is a faithful character of $\tilde{G}$ by Corollary 3.3 . With GAP one finds that there is only one faithful character $\chi_{W}$ of $\tilde{G}$ satisfying $\chi_{W} \otimes \chi_{W}=\tilde{\chi}$. The character $\chi_{W}$ contains the two complex conjugate irreducible characters $\psi_{1}$ and $\psi_{2}$ of degree 13 with multiplicity 1683 . Since $\operatorname{dim}(V) \equiv 2(\bmod 4)$ and $\mathbb{Q}\left[\psi_{1}\right]=\mathbb{Q}[\sqrt{-3}]$ Corollary 3.6 yields $d_{ \pm}(\varphi)=-3$. 


\section{Example 4}

The applications are not restricted to the characteristic 0 case. Let $V$ be the 4 -dimensional $\mathbb{F}_{2} A_{6^{-}}$ module. If $V$ admits a nondegenerate $A_{6}$-invariant quadratic form $q$, then there is a projective representation

$$
A_{6} \rightarrow C_{0}((V, q))^{*}
$$

yielding an irreducible $\overline{\mathbb{F}_{2}} A_{6}$-module of dimension 2 . Since there is no such module, one concludes that $V$ is not of quadratic type.

Now let $\varphi:=(V, q)$ be a 4-dimensional simple orthogonal $\mathbb{F}_{3} A_{5}$-module. Then there is a linear representation 2. $A_{5} \rightarrow C_{0}(\varphi)^{*}$ giving rise to a nontrivial action of $\overline{\mathbb{F}}_{3} 2 . A_{5}$ on the 2-dimensional simple $C_{0}(\varphi)$ module. Since the two irreducible $\overline{\mathbb{F}}_{3} 2 . A_{5}$-modules of dimension 2 are only realisable over $\mathbb{F}_{9}$, the determinant $d_{ \pm}(\varphi)=-1$ is not a square in $\mathbb{F}_{3}^{*}$.

\section{Example 5}

Let $G=U_{3}(5)$ and $\varphi:=(V, q)$ be a 21-dimensional simple orthogonal $\mathbb{Q} G$-module. The universal covering group of $G$ is $3 . G$. Therefore $P_{0}: G \rightarrow c(\varphi)$ can be chosen to be linear. There is a unique character $\chi_{W}$ of $G$ satisfying $\chi_{W} \otimes \chi_{W}=\tilde{\chi}$. In the notation of [Conway et al. 1985] one has $\chi_{W}=$ $2 \chi_{1}+\chi_{2}+2 \chi_{3}+2 \chi_{7}+2 \chi_{10}+\chi_{11}+\chi_{12}+\chi_{13}+\chi_{14}$. The character field of $\chi_{2}$ (of degree 20) is $\mathbb{Q}$ and its rational Schur index is 2. If $U$ is the irreducible $\mathbb{Q} G$ module with character $2 \chi_{2}$ then $\operatorname{End}_{\mathbb{Q} G}(U)=Q_{\infty, 5}$ the rational quaternion algebra ramified only at 5 and the infinite place. Now Corollary 3.6 yields $[c(\varphi)]=\left[Q_{\infty, 5}\right]$.

Let $q$ be a power of an odd prime $p$. As noted by a referee, the group $U_{3}(q)$ has a absolutely irreducible rational representation $V$ of degree $q^{2}-q+1$ (see [Simpson and Frame 1973]) and a rational character $\chi$ of degree $q(q-1)$ with Schur index 2 at $\infty$ and $p$ ([Gross 1990, Section 14]). So one might hope to generalize this calculation to arbitrary prime powers $q$. For $q=7$ the character $\chi$ occurs with odd multiplicity in $\chi_{W}$, so here $[c(\varphi)]=\left[Q_{\infty, 7}\right]$. If $q=3$ or $q=11$, then $\operatorname{dim}(V) \equiv \pm 1(\bmod 8)$. Therefore $\mathbb{R}$ splits $c(\varphi)$ for any positive definite quadratic form on $V$ and $\chi$ can not occur with odd multiplicity in $\chi_{W}$. But here one finds that the trivial character has odd multiplicity in $\chi_{W}$, hence $[c(\varphi)]=1$ in these cases. For $q=9$ the calculations do not allow to determine $[c(\varphi)]$ uniquely. It seems to be very difficult to calculate the candidates for $\chi_{W}$ (for $q=7,9,11$ one has two possibilities for the character $\left.\chi_{W}\right)$ generically.

As a referee pointed out, some of the examples above can also be considered from the integral lattice point of view, replacing the determinant by the determinant module $L^{\# / L}$, where $L$ is a $G$-invariant lattice in the $\mathbb{Q} G$-module $V$ and $L$ is integral, that is, contained in its dual lattice

$$
L^{\#}:=\left\{v \in V \mid B_{q}(v, L) \subseteq \mathbb{Z}\right\} .
$$

If $L$ is a maximal integral $G$-invariant lattice in $V$, then $L^{\#} / L$ is a direct sum of simple selfdual $\mathbb{F}_{p} G$ modules, where $p$ runs through the primes dividing $\left|L^{\#} / L\right|$. If all selfdual $p$-modular constituents of $V$ have even degree, then $p$ does not divide the determinant of $V$. This observation immediately yields that $\operatorname{det}(\varphi)=1$ in Example 1, since the character is absolutely irreducible modulo every prime $p$. Similarly the only primes $p$ that can divide $\operatorname{det}(\varphi)$ are 3 and 5 in Example 2 and 3 in Example 3. In Example 3 one even can conclude that $\operatorname{det}(\varphi)=3$, because otherwise $G$ would fix an even unimodular lattice $L \subset V$, and hence $8 \mid \operatorname{dim} V$.

\section{Conclusion}

This method is better called a trick, because it can not be applied too often. For instance, for the finite simple groups only the first two or three characters $\chi_{V}$ usually have a real chance that there is such a constituent $\psi$ of $\chi_{W}$ with odd multiplicity. But when this trick can be applied, the calculations are easy and it is surprising to see the determinant of a $G$-invariant quadratic form appear in the character table.

\section{ACKNOWLEDGMENTS}

I thank Prof. Carl Riehm for valuable questions and corrections on this paper.

\section{REFERENCES}

[Conway et al. 1985] J. H. Conway, R. T. Curtis, S. P. Norton, R. A. Parker, and R. A. Wilson, Atlas of finite groups, Oxford University Press, Oxford, 1985. 
[Fröhlich 1972] A. Fröhlich, "Orthogonal and symplectic representations of groups", Proc. London Math. Soc. (3) 24 (1972), 470-506.

[Gagola and Garrison 1982] S. M. Gagola, Jr. and S. C. Garrison, III, "Real characters, double covers, and the multiplier", J. Algebra 74:1 (1982), 20-51.

[Gross 1990] B. H. Gross, "Group representations and lattices", J. Amer. Math. Soc. 3:4 (1990), 929-960.

[Hasse 1924] H. Hasse, "Äquivalenz quadratischer Formen in einem beliebigen Zahlkörper", J. reine angew. Math. 153 (1924), 158-162.

[Jansen et al. 1995] C. Jansen, K. Lux, R. Parker, and R. Wilson, An atlas of Brauer characters, Oxford University Press, New York, 1995. Oxford Science Publications.

[Knus 1991] M.-A. Knus, Quadratic and Hermitian forms over rings, Grundlehren der Math. Wiss. 294, Springer, Berlin, 1991.

[Nebe 1999] G. Nebe, Orthogonale Darstellungen endlicher Gruppen und Gruppenringe, Aachener Beiträge zur Mathematik 26, Verlag Mainz, 1999. Habilitationsschrift RWTH Aachen.

[Nebe 2000] G. Nebe, "Orthogonal Frobenius reciprocity", J. Algebra 225:1 (2000), 250-260.

[Reiner 1975] I. Reiner, Maximal orders, London Math. Soc. Monographs 5, Academic Press, London, 1975.

[Scharlau 1985] W. Scharlau, Quadratic and Hermitian forms, Grundlehren der Math. Wiss. 270, Springer, Berlin, 1985.

[Schönert et al. 1994] M. Schönert et al., GAP: Groups, algorithms, and programming, 4th ed., Lehrstuhl D für Mathematik, RWTH Aachen, 1994. See http:// www-gap.dcs.st-and.ac.uk/ gap.

[Simpson and Frame 1973] W. A. Simpson and J. S. Frame, "The character tables for $\mathrm{SL}(3, q), \mathrm{SU}\left(3, q^{2}\right)$, $\operatorname{PSL}(3, q), \operatorname{PSU}\left(3, q^{2}\right)$ ", Canad. J. Math. 25 (1973), 486-494.

[Turull 1992] A. Turull, "The Schur index of projective characters of symmetric and alternating groups", Ann. of Math. (2) 135:1 (1992), 91-124.

Gabriele Nebe, Abteilung Reine Mathematik, Universität Ulm, 89069 Ulm, Germany (nebe@mathematik.uni-ulm.de, http://www.mathematik.uni-ulm.de/ReineM/nebe) 